\title{
Summary of Searches for SUSY in Photonic States and Long Lived Particles (CDF+D0)
}

\author{
Elemér NAGY (CPPM)
}

on behalf of the CDF and DØ Collaborations

\begin{abstract}
New results are presented in testing Supersymmetry at the Tevatron using photonic final states and searching for long-lived neutral and charged particles.
\end{abstract}

Keywords: Supersymmetry, Tevatron, CDF, D0, Photonic states, Long-lived particles

PACS: $12.60 .-\mathrm{i}, 12.60 . \mathrm{Jv}, 14.80 . \mathrm{Ly}, 29.20 . \mathrm{db}$

\section{INTRODUCTION}

Supersymmetry (SUSY) is a symmetry of Nature for the interchange of bosons and fermions. It is a basic ingredient of theories aiming at unification of all four interactions. In the minimal supersymmetric extension of the standard model (MSSM) every standard model (SM) particle has a SUSY partner differing its spin by $1 / 2$. Defining $R$-parity as $R=(-1)^{3 B+L+2 S}$, where $B$, $L$ and $S$ are the baryon number, lepton number and the spin, respectively, SM particles have $R=+1$, whereas the SUSY partners are characterized by $R=-1$. In the MSSM the Higgs sector consists of two doublets. In the present talk we assume $R$-parity conservation, which implies that the lightest SUSY particle (LSP) is stable.

If SUSY were exact only one additional parameter would be needed with respect to the SM: $\mu$ the Higgs mass mixing parameter. However SUSY is a broken symmetry since no SUSY partners of SM particles have been seen yet. The description of the symmetry breaking introduces a large number of parameters, which can be reduced by additional hypotheses. In the present talk we consider the so called gauge mediated supersymmetry breaking (GMSB) mechanism which has six parameters: $\Lambda$ - the supersymmetry breaking scale, $M_{m}$ - the mass scale of messengers which mediate the symmetry breaking to the electroweak scale, $N_{5}$ - the number of messenger fields which determines the nature of the next lightest SUSY particle (NLSP), $\tan \beta$ - the ratio of the vacuum expectation values of the two Higgs doublets, $C_{\text {grav }}$ - a factor which determines the lifetime of the NLSP and finally, the sign of $\mu$ [1]. In the GMSB model the LSP is the gravitino, the SUSY partner of the graviton.

\section{THE TEVATRON AND THE DETECTORS}

The Tevatron is a proton-antiproton collider producing collisions at $1.96 \mathrm{TeV}$ energy in the center of mass. It has delivered already more than $4 \mathrm{fb}^{-1}$ integrated luminosity. $8 \mathrm{fb}^{-1}$ integrated luminosity is expected if running in 2010 will be approved. Data are taken by two large detectors constructed and being operated by the $\mathrm{CDF}$ and $\mathrm{D} \emptyset$ Collaborations. In the present talk, with a single exception, results are based on data collected in the periode of RunIIa, corresponding to about $1 \mathrm{fb}^{-1}$. This means that more than an order of magnitude improvement in luminosity is expected for most of the analyses around 2010 by combining the results of the two collaborations.

The two detectors [2] have similar structure: a central tracker inside a solenoidal magnetic field is followed by a calorimeter with electromagnetic and hadronic compartments, surrounded by the detector for muons. For the present talk the central preshower (CPS) in the DØ detector is particularly important, because it helps to separate hadron jets from photons and together with the four layers of the electromagnetic compartment of the calorimeter it provides an efficient tool to determine the direction of photons. Timing information, necessary for searching long-lived particles is provided in the CDF detector at three places: by the TOF counters at the end of the tracking volume, by drift time measurements in the central tracker and by the timing system in the electromagnetic calorimeter. Similar information is obtained from the scintillating trigger counter in the $\mathrm{D} \varnothing$ detector. 

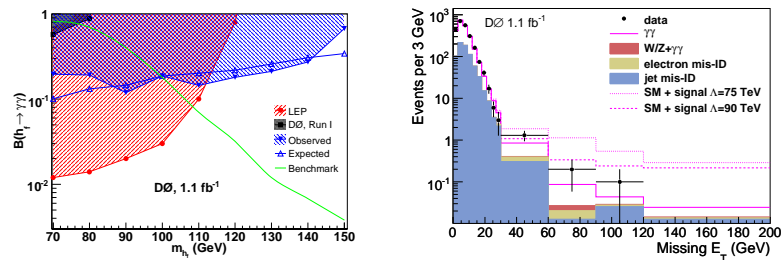

FIGURE 1. Left: Observed (full triangles) and expected (empty triangles) lower limits on the BR of fermiphobic Higgs particles as a function of the Higgs mass. Also shown are the LEP limits (full circles) and the theoretical prediction (solid line). Right: MET distribution observed in association with two high $p_{T}$ photons. The estimated backgrounds from SM as well as possible GMSB signals are also shown.

\section{RESULTS}

\subsection{Search for fermiophobic Higgs particles}

In some extensions of the SM the Higgs particle may not couple to fermions. This happens e.g. in the twodoublet models when the mixing angle between the two CP-even Higgs particles is $90^{\circ}$. In this case the branching ratio (BR) into two photons may be even close to 1 . $\mathrm{D} \emptyset$ has searched [3] for peaks in the invariant mass distribution of two photons of high transverse momentum $\left(p_{T}\right)$. In the absence of the signal a lower limit on the BR has been obtained, which in turn, allowed to exclude fermiophobic Higgs particles with mass below $100 \mathrm{GeV}$ (Fig. 1(left)).

\subsection{Search for GMSB}

\subsubsection{Neutralino NLSP}

If $N_{5}=1$, the NLSP is the lightest neutralino, the SUSY partner of neutral gauge bosons and Higgs particles. It decays to a photon and the gravitino. This latter escapes detection. The decay is prompt if $C_{\text {grav }}$ is near to 1 . The signature of such events are two high $p_{T}$ photons pointing to a primary vertex and missing transverse energy (MET). DØ has searched for such events making use of an efficient photon pointing algorithm which helped to select the correct primary vertex of the prompt photons. No significant excess has been found in the MET distribution beyond the SM prediction (Fig. 1(right)). This allowed to exclude $\Lambda<91.5 \mathrm{GeV}$ and thereby neutralinos with mass below $125 \mathrm{GeV}$ and charginos (the SUSY partners of charged gauge bosons and Higgs particles) with mass below $229 \mathrm{GeV}$ [4].
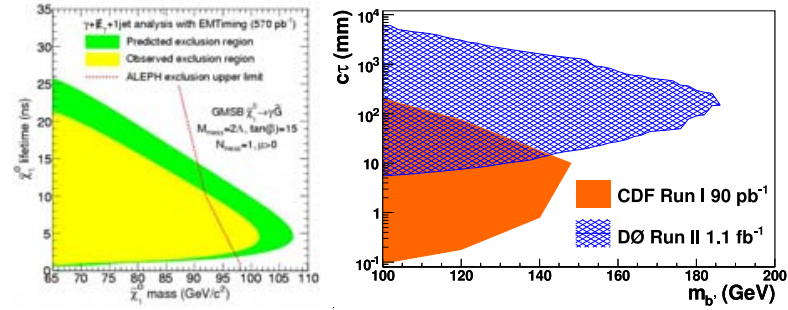

FIGURE 2. Left: Observed and expected excluded regions in the plane of the neutralino lifetime vs. its mass. Right: Excluded regions in the plane of a 4 th generation $b^{\prime}$ lifetime vs. its mass.

CDF has searched for events with long-lived neutralino NLSP $\left(C_{\text {grav }} \gg 1\right)$. In this case the photons arrive late in the calorimeter. The optimal time window for such events has been found $2<t_{c}^{\gamma}<10 \mathrm{~ns}$, where $t_{c}^{\gamma}$ is the difference between the time of flights of delayed and prompt photons. 2 events have been found in this time window whereas $1.25 \pm 0.66$ events have been expected from the collision (SM) and non-collision (cosmic muons and beam halo) background. This allowed to exclude a large domain in the plane of the neutralino lifetime versus its mass as shown in Fig. 2(left) [5].

The case of a long-lived neutralino NLSP decaying into a gravitino and a $Z$ boson was investigated both by $\mathrm{CDF}$ and $\mathrm{D} \emptyset$. The secondary vertex was reconstructed in the tracker by $\mathrm{CDF}$, whereas $\mathrm{D} \emptyset$ used the four layers of the electromagnetic calorimeter and the CPS. When the direction pointing from the primary to the secondary vertex coincided with that of the $Z$ boson, the distance between the two vertices was considered positive. For long-lived particles an excess of positive distances is expected. The data, on the other hand didn't show any such excess. This allowed to set limit on the lifetime of the long-lived particle vs. its mass. Fig. 2(right) shows an exclusion plot obtained for a 4 th generation $b^{\prime}$ quark [6].

\subsubsection{Stau NLSP}

If $N_{5}=3$, the NLSP may be an stau, the SUSY partner of the $\tau$ lepton. DØ searched for pair production of longlived staus [7] assuming $C_{\text {grav }} \gg 1$. These particles, commonly called charged massive stable particles (CMSP) appear in the detector as slowly moving muons, having speed significance of the order of 2-10, where this latter is defined as the difference of the speed of the light and that of the staus divided by the measurement error of the speed. Having selected two high $p_{T}$ muons with positive speed significance and applied veto against cosmic rays the final isolation of the signal was carried out on the plane of the invariant mass of the muon pair vs. the product of the speed significance of the two muons. The 

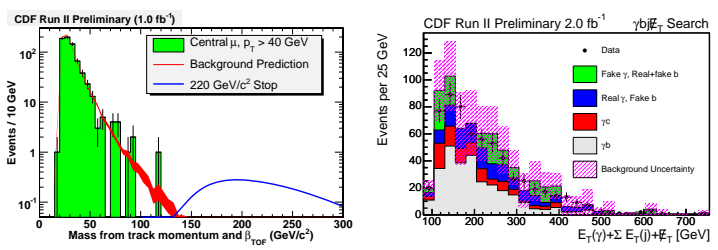

FIGURE 3. Left: Mass distribution obtained in the search for long-lived stop quarks. Right: The observed and expected distribution of the scalar sum of the transverse momenta of the photon $+b+j e t+M E T$ final states.

data was found to be compatible with the expected background, e.g. no events was observed above CMSP masses of $100 \mathrm{GeV}$ where the typical background amounts to $0.60 \pm 0.05$. Therefore, an upper limit of the cross section for long-lived stau production has been set. The small number of expected signal events didn't allow to transform this limit to limit on the stau mass. However, assuming that the CMSP is a long-lived chargino and the mass difference between the chargino and the neutralino is less than $150 \mathrm{MeV}$, the derived CMSP cross section limit has been used to exclude masses below $140 \mathrm{GeV}$ and 174 $\mathrm{GeV}$ for higgsino-like and for gaugino-like charginos, respectively.

\subsection{Search for long-lived stops}

CDF has searched for production of long-lived stops, the SUSY partners of the top quark. Two penetrating (muon-like) particles with transverse momentum $p_{T}>$ $40 \mathrm{GeV}$ and with $\beta<0.9$ have been selected, where $\beta$ is the relative speed of the particle with respect to the speed of light. The speed has been estimated as the ratio of the pathlength to the time of flight, measured by the TOF counters and cross checked with the time measurements in the drift chambers. The mass square $M^{2}$ of the particle has been calculated by $M^{2}=p^{2}\left(1 / \beta^{2}-\right.$ $1)$, where $p$ is the momentum. The background has been estimated by the convolution of the $p^{2}$ and $1 / \beta^{2}-1$ distributions of prompt particles of $p_{T}<20 \mathrm{GeV}$. As one can see in Fig. 3(left) the data agrees with the estimated background and there is no indication for the presence of the stop. This allowed to determine upper limit of the stop production cross section and exclude long-lived stops with a mass smaller than $250 \mathrm{GeV}$ [8].

\subsection{Search for anomalous production of a photon+b+jet+MET final state}

CDF has searched for events with a final state containing a photon, two jets (with one tagged as $b$-jet), ac- companied by significant MET. Such a final state cannot be easily produced by SM processes, whereas charginoneutralino pair production can give rise to it via $\chi_{1}^{+} \chi_{2}^{0} \rightarrow$ $(\bar{b} \tilde{t})\left(\gamma \chi_{1}^{0}\right) \rightarrow \gamma \bar{b} c M E T$. Fig. 3(right) shows the observed and expected distribution of the scalar sum of the transverse momenta. As can be seen, no excess beyond the expected SM background has been found [9].

\section{CONCLUSION}

Photonic final states and long-lived particles are powerful tools for searching new physics, in particular SUSY at the Tevatron. The excellent performance of the collider and detectors of the CDF and D $\varnothing$ Collaborations accompanied with innovative methods in the analyses allowed to study these interesting event topologies. No evidence for new physics has been found so far, thereby the parameter space for SUSY has shrinked considerably. More details can be found on the CDF and DØ web sites: http: //www-cdf . fnal.gov/physics/exotic/exotic.html, http://www-do.fnal.gov/Run2Physics/ WWW/results/np.htm.

\section{ACKNOWLEDGMENTS}

The help of the CDF and D $\varnothing$ Physics Coordinators and Physics Group Conveners, especially that of Todd Adams and Chris Hays, is highly appreciated.

\section{REFERENCES}

1. A detailed description of the SUSY phenomenology can be found e.g. in the ATLAS Detector and Physics Performance Technical Design report, Vol II. CERN/LHCC/99-15, ATLAS TDR 15, 25 May 1999.

2. A detailed description of the $\mathrm{CDF}$ and $\mathrm{D} \emptyset$ detectors can be found in D. Acosta et al. (CDF Collaboration), Phys. Rev. D 71, 032001 (2005) and in V.M. Abazov et al. (DØ Collaboration), Nucl. Instrum. Methods Phys. Res. A 565, 463 (2006), respectively.

3. V.M. Abazov et al. (DØ Collaboration), FERMILAB-PUB08/057-E, submitted to Phys. Rev. Letters

4. V.M. Abazov et al. (DØ Collaboration), Physics Letters B 659, 856 (2008)

5. A.Abulencia et al. (CDF Collaboration), FERMILABPUB-07-075-E, submitted to Phys. Rev. Letters

6. F. Abe et al. (CDF Collaboration), Phys. Rev. D 58051501 (1998)

V.M. Abazov et al. (DØ Collaboration), Fermilab-PUB08/169-E, submitted to Phys. Rev. Letters

7. V.M. Abazov et al. (DØ Collaboration), DØ Note 4746-CONF

8. CDF Collaboration, CDF Note 8701

9. CDF Collaboration, CDF/PUB/EXOTIC/PUBLIC/9296 